\title{
Status of the Streamer Mode in Gas Detectors
}

\author{
V. I. Razin \\ Institute for Nuclear Researches, Russian Academy of Sciences, Moscow, Russia \\ Email: razin@inr.ru \\ Received 17 February 2014; revised 17 March 2014; accepted 24 March 2014 \\ Copyright (C) 2014 by author and Scientific Research Publishing Inc. \\ This work is licensed under the Creative Commons Attribution International License (CC BY). \\ http://creativecommons.org/licenses/by/4.0/

(c) (i) Open Access

\section{Abstract}

This work shows the results of a streamer discharge mode studies in various gas detectors developed up-to-date. The results are based on a new experimental data from high-speed thin-gap gas detector application developments as well as on basic knowledge of multi-wire devices operations.

\section{Keywords}

\section{Gaseous Detectors, Micropattern Gaseous Detectors (MSGC, GEM, THGEM, RETGHEM, MICROMEGAS, RPC, etc.)}

\section{Introduction}

More than 40 years have passed since G. Charpak and F. Saulu [1] have discovered the high current mode of operation for multi-wire proportional chambers, which is known as "self-quenched streamer mode" (SQS) [2]. Nevertheless, there are a lot of unstudied features in the nature of this phenomenon in gases at normal pressure. The SQS-mode has been associated at the first time with large gas chambers and high fraction of extinguishing part (namely hydrocarbons and methylal) with thick anode wires. The existence of a high current mode has been discovered now in wireless thin-gap devices with position sensitivity (RPC, GEM, MICROMEGAS [3]-[5]) as well.

\section{Main Part}

The main features of the streamer discharge mode have been proved based on results of a new experimental data. The features have been observed in most of position sensitive gas detectors during the studies. These are:

1) There is a typical transition of a gas-discharge device to a streamer mode with a jump in the signal amplitude. Small and large signals exist simultaneously in the transition region. They are caused by the avalanche 
amplification mechanism and the streamer formation, respectively. There are several disjoint amplitude characteristic curves as well.

2) There is a basic difference in a shape of curve for an exponential avalanche multiplication with saturation as well as linear dependence of a charge increase in a streamer mode with the voltage applied.

3) The Raether's threshold is the necessary (but not sufficient) condition for the avalanche to streamer transition: a $\times \mathrm{N}_{0} \geq 10^{8}$, where "a" is the gain amplification in the avalanche and " $\mathrm{N}_{0}$ " is the initial number of electrons. It is known that a probability of a streamer is higher in gas mixtures than in pure gases.

4) A plasma is the most probable state at gas gain of about $10^{7}-10^{8}[6]$. The basic features are slowing down of electron, electric charge neutralization and electrostatic plasma oscillations of an ion branch.

5) Localization of a streamer occurs mainly near the drift direction of initial electrons and main avalanche axis, i.e. in the area of large number of excited and metastable atoms (dimers) and almost without any photo-ionization.

6) A streamer mode signal has a shape like a signal from a pulse ionization chamber.

7) Streamer formation process is always followed by a pulse-precursor, which has a sufficiently large amplitude compared to usual pulse from proportional mode. This pulse-precursor is connected to the secondary avalanche developing in the tiny area of a gas detector with a maximum density of a positive space charge. In other words, there are three types of pulses in SQS-mode:

a) proportional;

b) avalanche precursor;

c) streamer.

8) The plasma state can be transformed into a narrow luminous channel or a streamer in a detector gas volume as a result of a several electrostatic and electromagnetic interactions passed through the gas after the induced negative charge has been released from cathode [7].

9) The plasma state can be reached in the thin-gap gas discharge device with thickness of about $20 \mathrm{mkm}$. In this case, the electron can get energy for direct ionization in $100 \mathrm{kV} / \mathrm{cm}$ electric field according to $10^{6}$ approximately equal $2^{20}$.

10) Counting efficiency of streamer events is saturated w.r.t. the full number of events (streamer and proportional ones) and does not exceed a value of $50 \%$ after certain threshold voltage has been reached between the electrodes of the detector.

11) There is a time delay from a few nanoseconds to several hundreds of microseconds between the streamer signal appearance and the pulse-precursor. The delay is independent from types of gas discharge position sensitive devices [8].

\section{Conclusions}

A brief analysis of recent results shows the possibilities to determine the necessary and sufficient conditions for streamer formation in gas position sensitive detectors based on the SQS-mode. The plasma laws can be applied to study of this phenomenon with the whole chain including the pulse-precursor with higher amplitude w.r.t. proportional signal pulses.

The role of the pulse-precursor has to be studied for the secondary avalanche in the area of maximum density of positive space charge. One should take into account the effect of presence of slow electrons when the electric field strength is equal to the external one and the space between electrodes has a short circuit due to an elastic ionization wave. One should explain the sudden jump in pulse current with passing the induced negative charge from cathode resulting in a narrow luminous (from recombination) channel or streamer appearance.

The SQS-mode studies are stimulated by Micro-Pattern Gas Detectors (MPGD) widely used now because of their excellent spacial resolution as well as high counting rates. These detectors are well known as small photon feedback mechanism devices. Therefore they have a small probability of formation and propagation of streamers. The electric field is almost homogenous in MPGD because of the absence of wires. This makes the calculation simpler including so called basic localization discharge principle, i.e. any discharge in the gas gap should produce only a local electric field drop. In addition, MPDG has an electron multiplication at the holes and a step avalanche developing mode as well as a high gain operating without spark discharges. These features open a good challenge to study in details the streamer formation mechanism and transition to spark dis- 
charges.

\section{Acknowledgements}

Author thanks to my colleagues: Hliustin D.V. and Morozov S.V. for useful discussions.

\section{References}

[1] Charpak, G. and Sauli, F. (1971) Multiwire Chambers Operating in Geiger-Muller Mode. Nuclear Instruments and Methods, 96, 363-367. http://dx.doi.org/10.1016/0029-554X(71)90601-X

[2] Alekseev, G.D., et al. (1980) Investigation of Self-Quenching Streamer Discharge in a Wire Chamber. Nuclear Instruments and Methods, 177, 385-397. http://dx.doi.org/10.1016/0029-554X(80)90049-X

[3] Giomataris, Y., et al. (1997) MICROMEGAS: A High-Granularity Position-Sensitive Gaseous Detector for High Particle-Flux Environments. Nuclear Instruments and Methods in Physics Research, 306, 531.

[4] Chechik, R., et al. (2006) Advances in Thick GEM-Like Gaseous Electron Multipliers. Nuclear Instruments and Methods in Physics Research, 558, 475.

[5] Pestov, Yu.N. (2002) Review on Counters with Localized Discharge. Nuclear Instruments and Methods in Physics Research, 494, 447-454.

[6] Zalikhanov, B.Zh. (1998) Plasma Discharge Mechanism in Wire Chambers. Fizika Elementarnykh Chastits i Atomnogo Yadra, 29, 1194.

[7] Razin, V.I. and Reshetin, A.I. (2012) Features of Gas Discharge in Narrow-Gap Micropattern Gas Detectors at High Level of Alpha-Particle Background. Physics of Particles and Nuclei Letters, 9, 58-61.

[8] Fonte, P., Peskov, V. and Ramsey, B. (1999) The Fundamental Limitations of High-Rate Gaseous Detectors. IEEE Transactions on Nuclear Science, 46, 321. 